JUAN CARLOS ZULUAGA D.*

JULIO CRUZ**

MARGARITA MENESES ${ }^{* * *}$

Universidad de Caldas (Manizales, Colombia)

\title{
Medicalización, promoción de la enfermedad y disfunción sexual femenina
}

\author{
Medicalization, disease mongering and female sexual \\ dysfunction
}

\author{
Medicalização, promoção da enfermidade e disfunção \\ sexual feminina
}

\footnotetext{
* Sociólogo (Univalle), especialista en políticas culturales (Universidad de Barcelona), Magister en Investigación en Ciencias Sociales (Universidad de Buenos Aires). Profesor del Departamento de Antropología y Sociología de la Universidad de Caldas. ORCID oooo-0oo2-8015-3044 Correo electrónico: juanc.zuluaga@ucaldas.edu.co

** Sociólogo y Licenciado en Lenguas Modernas de la Universidad de Caldas. Magister (c) en Estudios Políticos. Coinvestigador. Correo electrónico: juliocruzjotace@gmail.com

*** Estudiante de Sociología de la Universidad de Caldas. Auxiliar de investigación. Correo electrónico: ednis204@ hotmail.com

**** Este artículo de reflexión hace parte del marco teórico-conceptual desarrollado colectivamente para el proyecto «Disfunción sexual femenina y promoción de la enfermedad: aproximación desde el análisis crítico del discurso (ACD)», adscrito y financiado por el Departamento de Antropología y Sociología de la Universidad de Caldas. Artículo de investigación recibido el 24-10-2016 y aceptado 16-03-2017.
} 


\section{Cómo citar}

Zuluaga D, J. C.; Cruz, J. y Meneses, M. (2018). Medicalización, promoción de la enfermedad y disfunción sexual femenina.

Revista CS. (24), 41-66.

DOI: http://dx.doi.org/10.18046/recs.i24.2308 
Resumen

Abstract

Resumo

El artículo propone una reflexión crítica, si se quiere subalterna, frente al discurso hegemónico tanto en el campo de las ciencias de la salud como en el campo político-económico imperante, en torno a los procesos de medicalización y farmaceuticalización en las sociedades occidentales, haciendo énfasis en lo que algunos investigadores han denominado "promoción de la enfermedad», ejemplificada en el presente texto en la disfunción sexual femenina. A partir de la revisión y análisis de artículos de actualidad científica y crítica sociológica, se argumenta sobre el proceso sociohistórico contemporáneo que ha devenido en una «cultura farmacéutica», al igual que sobre los agentes, dispositivos y estrategias que permiten su expansión y reproducción. Las cuestiones problematizadas en el documento tienen que ver con la producción de sentido y las prácticas asociadas a los procesos de salud y enfermedad, así como su orientación y definición desde el ámbito de poder establecido por la institución médica, la industria farmacéutica y su lucrativo negocio de medicamentos.

\section{PALABRAS CLAVE:}

Medicalización; Farmaceuticalización; Promoción de la enfermedad; Disfunción sexual femenina.

The article proposes a critical reflection, if you want to subordinate, in front of the hegemonic discourse both in the field of health sciences as in the prevailing political-economic field, around the process of medicalization and Pharmaceuticalization in western societies, emphasizing what has been called «disease mongering», exemplified in the case of female sexual dysfunction. From the review and analysis of current scientific articles and sociological criticism, it is argued abaut the socio-historical contemporary process that has become a «pharmaceutical culture» as well as agents, devices and strategies that allow their production and reproduction. The issues problematized in the document have to do with the production of meaning and practices associated with the 
processes of health and disease, as well as its orientation and definition from the field of power established by the medical establishment, the pharmaceutical industry and its lucrative drug business.

\section{KEYWORDS:}

Medicalization; Pharmaceuticalization; Disease mongering; Female sexual dysfunction.

O artigo propõe uma reflexão crítica, se quiser subalterna, diante do discurso hegemônico tanto no campo das ciências da saúde como no campo político-econômico imperante, em torno aos processos de medicalização e farmaceuticalização sociedades ocidentais, fazendo ênfase no que alguns pesquisadores denominaram «promoção da enfermidade», exemplificada no presente texto na disfunção sexual feminina. A partir da revisão e analise de artigos de atualidade cientifica e crítica sociológica, se argumental sobre o processo sócio histórico contemporâneo que deveio de uma «cultura farmacêutica», igual que sobre os agentes, dispositivos e estratégias que permitem sua expansão e reprodução. As questões problematizadas no documento têm a ver com a produção de sentido e as práticas associadas aos processos de saúde e enfermidade, assim como sua orientação e definição do âmbito de poder estabelecido pela instituição medica, a indústria farmacêutica e seu lucrativo negócio de medicamentos.

\section{PALAVRAS CHAVES:}

Medicalização; Farmaceuticalização; Promoção da enfermidade; Disfunção sexual feminina. 


\section{Introducción: sociedades medicalizadas}

A lo largo de la historia, la humanidad ha orientado sus prácticas económicas, sociales y políticas a la constante búsqueda del mejoramiento de las condiciones de existencia. La sociedad occidental moderna no es una excepción, por el contrario, podría decirse que constituye un punto alto del imaginario que persigue un ideal utópico en todos los ámbitos de la vida. Desde distintas versiones ideológicas y en diferentes momentos históricos se ha buscado la supuesta perfección en lo espiritual, político, social y cultural; y más recientemente en lo biológico, que incluye la definición de aspectos clave como la salud, la enfermedad y la vida bajo orientaciones dadas, entre otras disciplinas, desde la ciencia médica. «La salud perfecta» $\mathrm{O}$ la «Gran salud», según Sfez (2008), es uno de los soportes de la utopía de los años 2000 y subsiguientes, que tiende a imponerse como el único proyecto mundial.

A partir del siglo XVIII la medicina se expande paulatinamente hacia campos exteriores al de la salud, de manera que el médico se convierte en una autoridad social y el hospital en una institución de control y medicalización (Natella, 2008). Según Foucault, para esta época se da la inclusión del ser biológico en el ámbito del poder, constituyendo un fenómeno de la modernidad occidental al que denomina y define como biopoder, el cual habla del «conjunto de mecanismos por medio de los cuales aquello que en la especie humana constituye sus rasgos biológicos fundamentales podrá ser parte de una política, una estrategia política, una estrategia general del poder» (Foucault, 2006: 15). En otras palabras: la aplicación e impacto del poder sobre todos los aspectos de la vida a través del campo médico. La biopolítica está pues encaminada en última instancia a que las mentes de las personas se autoregulen (controlen) y determinen sus actitudes y prácticas de acuerdo con los presupuestos del poder hegemónico.

Si bien por cuestiones de espacio este artículo no apunta a reconstruir en detalle el proceso de medicalización, ${ }^{1}$ es claro que la consolidación de la medicina moderna como mecanismo de poder desde finales del siglo XIX, asociada a los avances de la biología y la química molecular, produce entre otras cosas que la atención se traslade del paciente a la enfermedad; este conocimiento cada vez más detallado del nivel celular y molecular derivó en el desarrollo de fármacos dirigidos básicamente a tratar los síntomas. Vale decir que el uso cada vez más intensivo de las terapias farmacológicas va aparejado con una reconfiguración del campo médico en el marco de la sociedad capitalista, en tanto establece un vínculo directo con la dimensión políti-

1. Sobre la constitución y desarrollo de este proceso ver: Capra, F. (1992); Foucault, M. (2003; 2012); Rosenberg, C. (1992). 
co-económica, convirtiendo a la industria farmacéutica en una fuente de poder en la misma medida que se da un crecimiento del mercado de las drogas (Capra, 1992).

Puede decirse que las sociedades occidentales se han convertido en el escenario de extensión de la medicalización, la cual debe ser entendida según Kishore como «la forma en que el ámbito de la medicina moderna se ha expandido en los años recientes y abarca diversos problemas que antes no eran considerados como entidades médicas» (como se cita en Rodríguez, 2010:4). En tal sentido, muchos aspectos de la condición humana que antes no eran considerados como condiciones médicas son ahora definidos y tratados como tal, y nombrados bajo categorías como la de enfermedad o desorden (Blech, 2009; Conrad y Leiter, 2004).

El desarrollo y definición de los conceptos de salud/enfermedad es un paso previo al proceso de medicalización, donde la actual construcción sociocultural de las enfermedades está ligada a la concepción de la salud perfecta, que se patenta en una cultura del «ser saludable» (Sfez, 2008). De tal forma, la medicalización implica, por un lado, la creación de un sentido social sobre las prácticas del cuidado y la potencialización de la salud: esta búsqueda de la perfección auspicia la generación de procesos de mejoramiento, perfeccionamiento y/u optimización, que hacen posible la existencia de un tratamiento aun en casos en los que no existe una enfermedad. De otra parte, el proceso de medicalización como fenómeno presente en el siglo XIX y radicalizado en la sociedad actual, está orientado desde una lógica que apunta a la extensión de la frontera entre lo normal y lo patológico, que en la modernidad tardía ha llevado a lo que se conoce como «creación de no-enfermedades», «venta de enfermedades», «patologización de la vida cotidiana» 0 «construcción social de enfermedades» (Blech, 2009; Pérez-Ciordia, 2011; Rodríguez, 2015).

Ahora bien, facilita este proceso de medicalización la creencia de nuestra sociedad en la ciencia, la tecnología, la racionalidad y el progreso, sustentando el rol social del profesional médico y del científico, quienes con su discurso y conocimiento, y a través de su prestigio, terminan por legitimarlo (Conrad y Leiter, 2004). No obstante, es un proceso inscrito en el ámbito sociocultural, dado que debe ser entendido como un proceso multiactoral de una unidad entre médicos, mercados, consumidores, científicos, medios de comunicación, poder estatal e industria farmacéutica. Dicha unidad constituye al mismo tiempo un dispositivo en el que confluyen varias fuerzas descentralizadas y con diferentes niveles de implicancia en la medicalización, donde cada actor asume un rol en la intervención frente al proceso (Rodríguez, 2015). Según Conrad (2005), en las últimas décadas se han operado cambios en el campo médico, considerando que el rol del profesional médico como agente principal en los procesos de medicalización se ha desplazado hacia los consumidores y los intereses del mercado, en donde la industria químico-farmacéutica adquiere un poder especial. 
La imagen actual del cuerpo, sustentada y reforzada por los medios de comunicación, es la de una máquina que debe ser tratada por los médicos y las medicinas. ${ }^{2}$ En tal sentido, resulta casi una obviedad decir que, bajo el amparo de la legitimidad social de la ciencia, los anuncios publicitarios han maximizado el consumo de medicamentos, al tiempo que han generado un condicionamiento del gusto del consumidor, permitiendo la creación de un amplio mercado farmacéutico (Capra, 1992). Para el logro de su objetivo la industria procura un acercamiento directo a los consumidores a través de los medios masivos de comunicación, que por medio de discursos contundentes sobre la enfermedad y su tratamiento dificultan la posibilidad de controversia y contribuyen a la formación de expectativas que están por encima de la realidad (Conrad y Leiter, 2004).

Lo anterior indica que con la profundización del proceso de medicalización la enfermedad pasa de ser una construcción social a ser un proceso industrial «que alimenta y utiliza el deseo de estar sano» (Rodríguez Díaz, 2008: 76), en tanto el direccionamiento político de la salud es articulado al poder de la industria farmacéutica y de algunos grupos de interés (Blech, 2009). Como se argumentará en páginas posteriores, son estos agentes los principales patrocinadores de la investigación científico-médica encargada de definir las nuevas patologías, así como los productores y promotores de tratamientos farmacológicos, ya no sólo referidos a procesos biológicos sino a aparentes desórdenes de tipo social; haciendo incluso innecesaria la existencia de una posible enfermedad para acudir a los medicamentos, dado que se desarrollan medicinas preventivas que se usan de acuerdo con un perfil genético, etario o vinculado a procesos normales de la naturaleza humana, convirtiéndonos paulatinamente en una «sociedad hipocondriaca sana» (Pérez-Ciordia, 2011).

El proceso de interiorización en los sujetos de la concepción y las prácticas asociadas a la salud y la enfermedad, definidas estas según los criterios de la industria farmacológica en connivencia con la institución médica, es lo que el concepto sociológico de la farmaceuticalización propone enfocar, ya que permite entender el proceso de medicalización más allá de lo médico, esto es, a través de la transformación de los procesos sociales de la vida cotidiana a partir de la perspectiva del tratamiento de las enfermedades, fomentada por la industria farmacéutica y promulgada a través de los medios de comunicación. En tal sentido, la tesis de la farmaceuticalización afirma que los productos farmacéuticos se han convertido

2. Esta visión del cuerpo-máquina tiene antecedentes en la perspectiva cartesiana del siglo XVII (Aguilar, 2010; Foucault, 2012), y aunque tuvo contradictores en el siglo XIX se mantuvo vigente como metáfora, y fue reforzada por otros discursos, como los provenientes de la física y sus desarrollos en termodinámica (Pohl-Valero, 2010). 
en parte de nuestro diario vivir, enlazando la economía y la política de producción farmacológica a la vida privada de los ciudadanos, creando con ello una «cultura farmacéutica» (Doran y Hogue, 2014).

En los siguientes apartados se intentará ampliar algunos de los aspectos fundamentales referidos a lo que ha sido denominado como «promoción de la enfermedad», dar claridad conceptual y develar algunas de las estrategias y dispositivos a través de los cuales la industria farmacéutica y otros agentes se han encargado de crear, promover y comercializar medicamentos para supuestas dolencias trasmutadas en patologías. Como caso paradigmático se tomará la disfunción sexual femenina, que para algunos investigadores es un ejemplo palpable de invención y promoción de una patología inexistente o cuando menos de dudosa legitimidad científica (Applbaum, 2006; Meixel, Yanchar y Fugh-Berman, 2015; Moynihan, 2005; Moynihan, Heart y Henry, 2002; Shankar y Subish, 2007; Tiefer, 2006). Se desarrollará una sucinta presentación de los discursos sobre la sexualidad femenina en la sociedad moderna para luego mostrar la manera en la que se configuran e instituyen en el campo médico y farmacéutico los distintos trastornos sexuales de las mujeres.

\section{La promoción de la enfermedad}

«El que no muestra no vende», reza un viejo adagio popular, refiriéndose a la manera como el comercio se ha desenvuelto desde sus orígenes. Y es que, como lo muestra la historia, quienes han vivido del tráfico de bienes han hecho esfuerzos descomunales para dar a conocer sus productos, por llevarlos a todos los confines del orbe y por ponerlos en el imaginario de sus potenciales compradores. Desde las ferias medievales hasta las formas más sofisticadas de publicidad contemporánea, no se han escatimado gastos intelectuales ni pecuniarios para lograr lo que Marx (1977) denomina la realización de la mercancía, el cierre del círculo de la producción capitalista. Esta dinámica publicitaria no ha sido ajena al campo farmacéutico, el cual ha amasado enormes fortunas a base de aumentar los rubros destinados a su aparato mercadotécnico, asumiendo de paso los medicamentos como cualquier producto del mercado.

Mientras la publicidad se ha preocupado tradicionalmente por mercancías, la propaganda se ha concentrado en la comunicación de las ideas, por ejemplo, políticas. De acuerdo con Chomsky (2009: 12), una herramienta ideológica para «fijar los términos del discurso». Pero lo que se logra observar con la publicidad de los medicamentos, en lo que respecta al fenómeno conocido como «promoción de la 
enfermedad», es una confusión entre propaganda y publicidad, asunto delicado para la sociedad y de obligatoria atención para las ciencias sociales.

A continuación se verán los principales elementos constituyentes de la promoción de la enfermedad, sus estrategias y los alcances sociales que tiene, todo ello atravesado por el concepto de propaganda tal como es entendido en la actualidad, en relación con las élites estatales y los grupos de poder.

\section{Panorama de la promoción de la enfermedad}

El término «promoción de la enfermedad» fue acuñado por primera vez por Lynn Payer durante la década del 90 (Moynihan y Henry, 2006; Shankar y Subish, 2007), haciendo alusión a una práctica llevada a cabo por la institución médica, encarnada en las poderosas farmacéuticas, algunos médicos asociados a ellas y líderes de opinión (políticos entre ellos). Consiste en hacer creer a una persona que está enferma o que posee una enfermedad, a partir de argucias como la invención de una patología, la exageración de una dolencia o la transformación de una actividad natural en padecimiento (Doran y Hogue, 2014; Moynihan y Henry, 2006; Moynihan et al. 2002; Shankar y Subish, 2007). «La promoción de la enfermedad puede tornar dolencias ordinarias en problemas médicos, hacer ver síntomas leves como serios, tratar problemas personales como problemas médicos, ver riesgos como enfermedades» (Moynihan et al., citados en Shankar y Subish, 2007:275) [Traducción propia]. Es, en síntesis, «la venta de patologías que exceden las barreras de la enfermedad y aumentan los mercados de aquellos que venden y distribuyen tratamientos» (Moynihan y Henry, 2006: 01) [Traducción propia].

El antecedente teórico del concepto de promoción de la enfermedad viene dado por Ivan Ilich, quien, según Shankar y Subish (2007), explicó hacia la década del 70 el fenómeno basado en la expansión de la medicina hacia la vida de los individuos con el nombre de «medicalización». En Némesis Médica (1975), Ilich menciona una práctica polémica consistente en entregar la responsabilidad social de la salud de los individuos en la institución médica, en sus profesionales, teorías y medicamentos, asumiendo un control cada vez más grande sobre la salud de las personas, convirtiéndolas en pacientes. Existe un debate entre los expertos acerca de las diferencias entre la medicalización y la promoción de la enfermedad. Mientras que para algunos ambos conceptos son similares, para otros la diferencia radica en la forma más definida de la segunda en lo que respecta a sus intenciones económicas, e incluso hay quienes defienden las virtudes que la promoción de la enfermedad puede tener la para salud pública. Pero en general, se considera que «la promoción de la enfermedad 
es una "variante de la medicalización particularmente perniciosa, cínica, extrema y sucia"» (Doran y Hogue, 2014: 7) [Traducción propia].

El contexto que rodea la promoción de la enfermedad, se parece más al departamento de publicidad de cualquier empresa privada que a una organización que se encarga de atender la demanda de medicamentos de los ciudadanos con el fin de mejorar su salud y su calidad de vida. Las compañías farmacéuticas están dotadas de un aparato mercadotécnico que se encarga de aplicar estrategias para poner en el mercado los medicamentos, aun cuando no son requeridos; aumentar el número de sus potenciales consumidores, y hacer de las drogas un producto de consumo cotidiano (Doran y Hogue, 2014; Moynihan y Henry, 2006; Moynihan et al., 2002; Shankar y Subish, 2007). En un contexto económico competitivo los medios de información de masas son acaparados por las élites privadas, con el resultado de desequilibrar a su favor las relaciones de poder a través de la propaganda: «Un modelo de propaganda pone el énfasis en esta desigualdad de riqueza y poder, así como en los efectos que ésta produce a diferentes niveles en los intereses y elecciones de los medios de comunicación de masas» (Chomsky y Herman, 2009: 21-22).

El diseño de las campañas publicitarias no tiene nada que envidiar a las realizadas para las grandes firmas multinacionales o los candidatos políticos. Cuentan con líderes de opinión pagados por las farmacéuticas o con vínculos contractuales con ellas para que desde su posición social promuevan la adquisición de determinado producto farmacéutico, apelan a la presión ejercida a parlamentarios o políticos influyentes para que los controles a las drogas que producen sean mínimos, trabajan en anuencia con importantes periodistas y medios de comunicación que promocionan sus productos y hacen advertencias sobre las supuestas enfermedades que puedan estarse padeciendo, e incluso llegan a conformar asociaciones de usuarios o a apoyar grupos activistas que reclaman cierto tipo de medicamentos como un derecho (Applbaum, 2006; Blech, 2009; Meixel et al. 2015).

Una de las consecuencias más relevantes de la promoción de la enfermedad, en su búsqueda por ampliar su mercado y colonizar la vida de los potenciales compradores, es lo que se denomina el tránsito del «paciente-pasivo» al «consumidor-activo» (Doran y Hogue, 2014). Esta idea está sustentada en la dinámica económica contemporánea, en el individualismo metodológico y en la teoría de la elección racional (Paramio, 200o), la cual sostiene que el comprador posee la información suficiente y necesaria para satisfacer plenamente sus apetitos de consumo en el mercado, por lo que tanto el uno como el otro deben permanecer lo más libres posible. En este orden de ideas, el escenario médico se transforma en mercado farmacéutico de tal forma que el individuo deja de ser sujeto pasivo de la prescripción del especialista a con- 
vertirse en agente activo que busca de manera libre en el mercado los medicamentos que contribuirán a mejorar su calidad de vida y a lograr sus expectativas de salud:

El aumento de la mercantilización de la salud ha llevado a que los tratamientos médicos se tornen bienes comunes sujetos a las fuerzas del mercado, alentándonos a redefinir nuestra propia percepción de sujetos pacientes a consumidores activos. Mientras la transformación del paciente en consumidor puede ser empoderadora y una ganancia moral, también puede tener efectos adversos para la salud. Las personas saludables se consideran a sí mismas enfermas, toman drogas que no necesitan, experimentan efectos secundarios y pagan los costos por la medicación sin ningún beneficio. Mientras un diagnóstico puede beneficiar a aquellos que están genuinamente enfermos, la creación de «pacientes» que no están realmente enfermos puede crear ansiedad y efectos secundarios debido a los tratamientos, generando de este modo enfermedades genuinas sobrepasando cualquier valor futuro (Doran y Hogue, 2014: 12) [Traducción propia]

Esta proterva lógica, en la que los aspectos más delicados de la salud individual están puestos en manos de actores externos con el poder para tergiversar la realidad en su favor y en contra de los ciudadanos, está influida por un contexto económico que prioriza la ganancia privada sobre el interés general, una economía de mercado que se sustenta sobre la idea de que la persecución de la satisfacción personal devendrá inevitablemente en beneficio social, sobre la base de la «mano invisible» que pregona el individualismo metodológico (Paramio, 200o).

Una economía en la que lo fundamental es la conquista de mercados antes que la pertinencia de los productos, en la que la concreción de las transacciones comerciales prima sobre la resolución de problemas sociales acuciantes, en la que la maximización egoísta de la ganancia está por encima de las consideraciones altruistas: tal es el escenario para que las empresas farmacéuticas pongan en práctica cuanto recurso tengan a mano para expandir su negocio. Lo peligroso es que con ello expanden también la idea de enfermedad a regiones que antes no había habitado, logrando con ello que el consumo de medicamentos se torne un estilo de vida (Applbaum, 2006; Lexchin, 2006; Moynihan, Henry, 2006; Shankar y Subish, 2007).

\section{Estrategias de la promoción de la enfermedad}

Entre la diversidad de formas en que las empresas farmacéuticas logran hacer efectiva la promoción de la enfermedad, se cuentan aquellas que crean un ambiente científico y académico, ergo serio y riguroso, en torno a la mercantilización 
de las drogas. También se incluyen las formas de cooptar los grupos de interés de una manera u otra relacionados con el negocio de los medicamentos. Así mismo, la agitación de las supuestas enfermedades, sus factores de riesgo y las medicinas que servirían para curarlas, se hace a través de los medios de comunicación masivos, parte fundamental de estas estrategias de mercado. «La industria farmacéutica es una de las más rentables del mundo, y su inversión en publicidad [...] es mucho mayor que en la investigación y desarrollo de nuevos medicamentos» (Ramalho, 2010a: 121) [Traducción propia].

Los departamentos de publicidad y mercadotecnia de las grandes farmacéuticas destinan grandes sumas de dinero a promover estados que con una previa modificación de sus características puedan aparecer ante el gran público consumidor, y ante el público médico especializado, como enfermedades (Moynihan, 2005; Ramalho, 2010a;2010b; Shankary Subish, 2007). Después se relaciona esta enfermedad creada con un producto farmacéutico, supuestamente desarrollado con el fin de atender dicha condición (Meixel et al. 2015).

Las tres estrategias para «fomentar la creación de una condición y alinearla con un producto» son: elevar la importancia de una condición existente; redefinir una condición existente para reducir su estigma; desarrollar una nueva condición para construirle reconocimiento a una necesidad desconocida del mercado (Parry, 1998; como se cita en: Meixel et al., 2015: 859) [Traducción propia].

Como ejemplos ilustrativos de estas tres estrategias corporativas, estos autores señalan tres casos bastante ilustrativos y claros: el primero correspondiente a la elevación de la importancia de una condición ya existente para hacerla ver como enfermedad, ejemplifica el caso de la transformación de la acidez en la enfermedad denominada «reflujo gastro-esofágico», atendida farmacéuticamente con Zontac (Ranitidina), medicamento desarrollado por la farmacéutica británica Glaxosmithkline. El segundo caso es protagonizado por la empresa norteamericana Pfizer, que aplicando estrategias de mercado redefinió la impotencia sexual en «disfunción eréctil», eliminando así el estigma social que sobre ella existe, y despejando el camino para su tratamiento con Viagra (Citrato de Sildenafilo). La tercera estrategia, referida a la creación de una nueva condición, se ejemplifica en el denominado «desorden de deseo sexual hipoactivo», supuesta patología que alude a la disminución de la libido en las mujeres, cuyo ámbito de producción detallaremos en el apartado final de este documento.

Una vez se configura la transmutación de un estado natural o de una condición menor en enfermedad; o bien se amplía la prevalencia de un trastorno ya conocido; 
o se redefine una nueva condición devenida en patología. En todos los casos, la industria farmacéutica procura llevar este desarrollo al público receptor, tanto a los prescriptores como a los potenciales consumidores. Para ello trabaja dos frentes: Uno es la fuerte campaña publicitaria en medios de comunicación, donde se hace labor «pedagógica» en torno a la condición devenida en enfermedad, y paralelamente, por supuesto, se promociona la «solución», esto es: el medicamento correspondiente. El otro frente es una campaña de cooptación de los profesionales de la salud para «actualizarlos» en relación con esta nueva condición, preparándolos para recetar la droga en cuestión en sus consultas (Blech, 2009; Doran y Hogue, 2014). Para esto se llega al extremo de desarrollar centros de estudio de las supuestas enfermedades, crear foros académicos y cursos de actualización con expositores médicos que en la mayoría de las ocasiones tienen relaciones contractuales con las farmacéuticas (Blech, 2009; Meixel et al., 2015). «Los laboratorios farmacéuticos no se limitan a hacer publicidad de sus fármacos, sino que además promocionan las enfermedades a las que se dirigen» (Casino, 2006: 1).

Finalmente, el escenario está dispuesto para llevar el producto al destinatario final: al paciente devenido consumidor. Como ya se ha dicho, este consumidor de medicamentos adolece ahora de unas características que lo hacen más proclive a la publicidad de medicamentos, de paso es más funcional a la estrategia de aumento de mercado de drogas de las farmacéuticas, toda vez que ya no espera a que el médico le recete una medicina, sino que él mismo, de manera «autónoma», busca la que le haga falta para adecuar su estilo de vida a las exigencias de la sociedad de consumo, incluyendo en esta idea las (no) necesidades estéticas, físicas, mentales, preventivas, etc. (Blech, 2009; Doran y Hogue, 2014; Moynihan y Henry, 2006; Moynihan et al., 2002; Ramahlo, 2010a; 2010b; Shankar y Subish, 2007).

Apelando nuevamente a los postulados de Chomsky y Herman (2009) en relación con el modelo de propaganda de que hablan en su obra, es dable observar que este se ajusta de manera bastante satisfactoria con las acciones emprendidas por las compañías farmacéuticas en lo tocante a la promoción de la enfermedad:

Los ingredientes esenciales de ese modelo propagandístico o conjunto de nuevos «filtros» se engloban en los siguientes epígrafes: 1) La envergadura, la concentración de propiedad, la riqueza del propietario, y la orientación de los beneficios de las empresas dominantes en el ámbito de los medios de comunicación; 2) la publicidad como fuente principal de ingresos de dichos medios; 3 ) la dependencia de los medios de la información proporcionada por el gobierno, las empresas y los «expertos», información, por lo demás, financiada y aprobada por esos proveedores principales y por otros agentes del poder (22). 
Estos tres puntos de la disertación de Chomsky y Herman son suficientes para ilustrar la relación licenciosa entre los medios de comunicación privados y las empresas farmacéuticas. Efectivamente, el poder económico de estas últimas es enorme y, como ha quedado constatado, una parte considerable de dicho poder va encaminado a tener presencia en los medios publicitarios; al punto que este renglón de su actividad empresarial ha desplazado incluso al mismo desarrollo e investigación farmacéutica. Es innegable, además, la dependencia de los medios a este poder, tanto como fuente de información como por la costosa pauta publicitaria que ofrece. Este connubio crea un peligroso entorno para los pacientes-consumidores, sometidos al poder omnímodo de farmacéuticas y medios de información; sin mencionar que también las farmacéuticas destinan rubros a ejercer presión sobre los políticos a cargo de decisiones importantes sobre los medicamentos y los posibles controles que puedan evitar (Blech, 2009); así como para la creación o influencia sobre grupos activistas que demanden cierto tipo de medicamentos, como ocurrió con la pastilla Addyi, que promete aumentar la libido en mujeres que aparentemente sufren alguno de los trastornos que hacen parte de las patologías consideradas como «disfunción sexual femenina» (Meixel et al., 2015).

\section{Efectos y alcances sociales de la promoción de la enfermedad}

Varias son las enseñanzas que deja este abordaje de la promoción de la enfermedad como categoría de análisis. Una de ellas tiene que ver con la fuerte vinculación que existe entre ella y el fenómeno del consumismo que tanto aqueja a las sociedades modernas. Así como el consumismo pone el énfasis en bienes y servicios que no resultan esenciales para la supervivencia del individuo-y cuya actividad desplaza a la producción como la más importante en las sociedades modernas (Bauman, 2007)-, de igual manera, la promoción de la enfermedad tiene por efecto colocar las drogas en el mercado antes que preocuparse por desarrollar medicamentos que atiendan verdaderas necesidades para la salud pública (Blech, 2009; Ramahlo, 2010a; 2010b; Shankar y Subish, 2007).

De acuerdo con Bauman (2007) el consumismo es un fenómeno social contrario al consumo individual. Se constata en el hecho de que el estilo de vida basado en el consumo de drogas sin prescripción médica, el auge de la publicidad sobre medicamentos (Chomsky y Herman, 2009), y el desdén de los individuos frente a los profesionales de la salud, va en aumento de manera generalizada (Moynihan et al., 2006). 
La popularidad de los fármacos conlleva una familiaridad peligrosa con ellos. La promoción de la enfermedad coloniza ámbitos que anteriormente le parecían vedados, como la medicina alternativa, la de inspiración oriental, e incluso el terreno estético (Doran y Hogue, 2014). Cada vez es más frecuente encontrar publicidad relacionada con estos aspectos de la salud, y cada vez son más las personas que acuden a estas formas, buscando allí una salida al desprestigiado sistema de salud tradicional. El truco en este caso es mostrar estas dimensiones de la vida como parte del andamiaje publicitario de la promoción de la enfermedad, hacer retoques estéticos para mejorar la salud, y sumar al arsenal de estrategias mercadotécnicas una pléyade de prácticas de ejercitación y meditación que posibilitan una expansión mayor del mercado.

Frente a este fenómeno se ha abierto una honda discusión, tanto en el terreno académico como en el político (Dovan y Hogue, 2014; Mintzes, 2006; Moynihan et al., 2002; Shankar y Subish, 2007), alrededor de la pregunta si los gobiernos deben intervenir en este asunto, y si la academia tiene la responsabilidad de denunciar estas prácticas. Está abierta la discusión ética en torno a la promoción de la enfermedad. Una corriente crítica trata de desvelar los intereses económicos y las nefastas consecuencias que acarrea para la sociedad en su conjunto; para ello apela a las herramientas científicas a su alcance.

\section{Discursos modernos sobre la sexualidad femenina}

Si bien a lo largo de la historia en sociedades patriarcales como la nuestra se ha mantenido bajo vigilancia y control la sexualidad femenina, los mecanismos y las estrategias de control varían y se complejizan según cada momento histórico y contexto sociocultural. El desarrollo científico tecnológico de las sociedades modernas en Occidente a partir del siglo XIX, fundamentalmente en el campo de la medicina en su perspectiva biomédica y su orientación hacia la biología molecular, produjo descubrimientos tales como las sustancias químicas presentes en el sistema endocrino, y orientó en buena parte el proceso de construcción de las nociones de diferencia sexual, iniciando una nueva etapa de definición y resignificación de la sexualidad femenina (Rohden, 2008).

Con el descubrimiento de las hormonas se generó la naturalización de la diferencia sexual a través de una lógica médica de sustancialización, la cual provee los modelos explicativos de la economía del cuerpo de la mujer (Blech, 2009). Si durante casi todo el siglo XIX los cuerpos femeninos parecían gobernados por los 
ovarios, señalándolos como causantes de variopintas «disfunciones» del organismo de la mujer y, por tanto, proclives a ser extraídos; a principios del siglo XX esta condición pasa a ser ocupada por las sustancias hormonales, las cuales pasaron a considerarse fundamentales no solo en la explicación de los problemas físicos sino también mentales de las mujeres.

Tal como señala Rohden (2008), estos desarrollos en la química corporal configuraron el paso del razonamiento del exceso que envolvía la relación de los ovarios con el cerebro y la actividad sexual, a otra dominada por el déficit o la falta de fluidos hormonales, los cuales en principio se pensaba eran distintivos en los cuerpos de hombres y mujeres. Junto a estos desarrollos, hacia la segunda mitad del siglo pasado, se presenta en el mundo occidental aquello que Béjin (1987) denomina el proceso de racionalidad de la sexualidad, generado y estimulado en buena medida por discursos científicos de la sexología y el psicoanálisis, acogidos por discursos políticos de algunos sectores del movimiento feminista bajo ideales de independencia, liberación sexual e igualdad en la intimidad, los cuales visualizaban justamente a las mujeres como sujetos autocontrolados y autónomos. Estos discursos tenían correspondencia con el lenguaje sexológico que señalaba semejanzas entre hombres y mujeres en cuanto a potencia sexual, aun cuando en realidad se presupuestaba -según Trebisacce (2015)- una reconfiguración de la asimetría entre los géneros, estimulando la complementariedad heterosexual y el amor en pareja, destacando que el hombre guía a la mujer en su búsqueda de placer.

Si bien la sexología comienza a mediados del siglo XIX, solo a mediados del siglo XX se consolida y alcanza mayor repercusión, a partir de estudios como los de A. Kinsey, W. Masters y V. Johnson (Bullough, 1994). A partir de allí se generan innúmeras investigaciones, así como proliferan charlas, conferencias y difusión informativa sobre la sexualidad en los medios de comunicación; los cuales decantaban hacia el público los descubrimientos y las narrativas modernas sobre el sexo (Trebisacce, 2015).

Dichos discursos de la sexología están íntimamente imbricados con los discursos psicoanalíticos que para la época habían logrado incorporar la sexualidad en la imaginación moderna. Según Illouz (2007), el psicoanálisis sirvió de puente entre la medicina y prácticas especializadas como la psicología, la neurología y la psiquiatría, así como entre la cultura alta y la baja.

Puede decirse que tanto el discurso de la sexología como el del psicoanálisis configuraron para su época un «régimen de verdad» (Foucault, 2012) con pretensiones de legitimidad para discernir lo normal y lo patológico en lo que a la vida sexual refiere. Estos discursos estaban prioritariamente orientados a develar la verdad sobre el problema de la obtención de placer, aunque «podría precisarse que la "verdad" 
que realmente obsesionaba a todos estaba circunscrita a la sexualidad femenina» (Trebisacce, 2015: 55). Tanto así que para Masters y Johnson (1972) la década de los años 60 fue catalogada como la época de la preocupación orgásmica.

La sexología de los años 70 se distancia paulatinamente de los supuestos psicoanalíticos, alegando que los problemas sexuales de las mujeres eran de orden conductual, y la solución estaría relacionada con el conocimiento del cuerpo femenino y la reeducación sexual tanto para varones como para mujeres. En años posteriores, al tiempo que se marginalizaba la perspectiva psicoanalítica, se consolida la perspectiva clínica, tanto en su versión psicológica como la médica, y con ello se institucionaliza el campo sexológico de los años 8o, estimulado por los desarrollos en medicina bioquímica, endocrinología, urología, ginecología y farmacología (Jones y Cogna, 2012).

En los años 60 las perspectivas genéticas y neurológicas reforzaron la lógica dualista de la diferencia sexual e instaron a construir una imagen de la mujer a partir de los discursos sobre el cuerpo hormonal, genético o fisiológico, en donde se presenta el organismo femenino como gobernado por las transformaciones hormonales propias de sus estados cíclicos, tales como la menstruación y la menopausia (Blech, 2009), reafirmando así constantemente las diferencias y distinciones esenciales entre los sexos. Según Rohden (2008), las secreciones hormonales son fundamentales para el funcionamiento del organismo femenino y para su equilibrio mental, reafirmando el axioma que señala como causa de muchos comportamientos de las mujeres a los productos bioquímicos del sistema endocrino.

Con base en estas teorías reduccionistas, para la década de los 80 del siglo pasado la naturaleza del sexo empieza a ser investigada por una nueva «medicina sexual» creada por especialidades médicas como la urología, gracias a las tecnologías científicas y al apoyo significativo de las publicaciones científicas, pero sobre todo al auspicio económico de la industria farmacéutica. Entre los años 80 y 90 estos estudios se focalizaron en primera instancia en el organismo masculino, diagnosticando disfunciones como la baja del deseo, la inadecuada excitación o erección y la precocidad o retraso en la eyaculación o en el orgasmo (Tiefer, 2006). En la definición y diagnóstico de estas emergentes patologías tomaron papel activo las farmacéuticas que competían por orientar la práctica médica hacia el uso de fármacos. El mayor logro que estas alcanzaron fue la puesta en el mercado del Sildefanil (Viagra), por la farmacéutica Pfizer a finales de los 9o, para combatir la recientemente denominada disfunción eréctil, configurando así una corriente medicalizadora que empezaba a buscar mercado en las llamadas disfunciones sexuales femeninas. 


\section{Invención y Farmaceuticalización de la Disfunción Sexual Femenina}

Solo hasta 1997 los urólogos empiezan a hablar de la «disfunción sexual femenina» (DSF), haciendo diferencia entre la función sexual saludable y el trastorno médico. Ese año, en la ciudad de New York, con el patrocinio de nueve compañías farmacéuticas, tuvo lugar el primer encuentro de especialistas médicos reunidos para determinar el perfil clínico de la DSF. «El objetivo de la reunión era diseñar la estrategia adecuada para crear una nueva patología en función de los intereses económicos de la industria farmacéutica» (Forcades, 2006: 6).

En 1998 se celebró en Boston la primera conferencia internacional destinada a crear consenso en torno a la definición de la nueva patología. Dicha conferencia fue igualmente patrocinada por la industria farmacéutica, y 18 de los 19 conferencistas reconocieron tener vínculos con las empresas patrocinadoras u otras empresas de fármacos (Forcades, 2006). Al tiempo que se desarrollaban estas reuniones de especialistas se generaron los procesos de investigación clínica que sirvieran de soporte para la legitimidad científica y pública de la patología recién definida, publicitando entre médicos y clínicos su existencia a través de seminarios y cursos de educación médica continuada (Meixel et al., 2015).

En 1999 Laumann y otros autores (2000) publicaron el artículo «Disfunción sexual en EE.UU.: prevalencia y variables predictivas», concluyen que el $43 \%$ de la población femenina de Estados Unidos sufría disfunciones sexuales definidas según los intereses de la industria farmacológica; sin embargo, se presentó un generalizado y creciente desacuerdo científico tanto en la definición de las patologías como en su prevalencia. Además, los beneficios de medicamentos experimentales para dificultades sexuales de las mujeres fueron cuestionados, así como los conflictos de intereses económicos de los expertos que apoyaban la noción de una condición médica muy prevalente (Moynihan, 2005).

Con base en el modelo masculino, los trastornos diagnosticados para algunas mujeres fueron clasificados como: a) trastornos del deseo; b) trastornos de la excitación; c) trastornos del orgasmo; d) trastornos dolorosos o «dispareunia»; y e) trastornos ginecológicos, enfermedades o dificultades en los órganos sexuales y reproductivos. Los críticos no niegan la existencia de estos trastornos, la gravedad de los síntomas o el valor del tratamiento médico para algunas mujeres enfermas. Lo que argumentan es que cada uno refleja una ampliación de los límites de la enfermedad en su definición y prevalencia, que finalmente permite y legitima la intervención médica y consolida la expansión del mercado para productos nuevos o prescriptos para otras patologías (Doran y Hogue, 2014). 
Según Tiefer (2006) y otros autores, la creación de la DSF es un claro ejemplo de «promoción de la enfermedad» por parte de la industria farmacéutica y de otros agentes de la medicalización, pues como se reseñó en páginas anteriores las compañías farmacéuticas no son en absoluto los únicos agentes involucrados, aunque sí los más influyentes. A través de la labor de investigadores críticos y periodistas de investigación se han develado las alianzas informales entre corporaciones farmacéuticas, empresas de relaciones públicas, grupos médicos y asociaciones defensoras de pacientes, los cuales trasladan estas ideas a la opinión pública y a los responsables de decisiones políticas, a menudo utilizando medios de comunicación para presentar e imponer una visión sobre un problema de salud en particular (Moynihan y Henry, 2006).

En la medida en que se logre hacer creer a las mujeres de sufren una disfunción o ausencia de determinadas sustancias químicas, estas querrán acercarse a los tratamientos científicos, para beneficio de la industria farmacológica. Gracias a esto, en los últimos años se ha generalizado el uso de productos para la DSF, como la benzodiacepina y el risedronato, inicialmente prescriptos para el tratamiento de otros trastornos, junto con sustancias de origen hormonal, como la testosterona y el estradiol (estrógeno), con los cuales se pretende el aumento de la líbido y la excitación o el alcance de mayor placer y satisfacción en las relaciones sexuales.

Tal como señala Tiefer (2006): si bien en las publicaciones científicas sobre el tema destacan aspectos psicológicos de la DSF, terminan por acentuar las implicaciones de los flujos químicos según los estados «normales» de los órganos genitales. La medicina biomédica reconoce factores de índole psico-mental, socio-relacional, ecológico y cultural como mediadores en la respuesta sexual humana (RSH), pero se presenta como hegemónica la visión neurofisiológica en la práctica médica, según la cual «el papel de las hormonas y neurotransmisores es determinante para entender la RSH y muchas de las disfunciones sexuales que pudieran aparecer» (Antona, 2013:9)

Los intentos de medicalización de la disfunción sexual femenina generan intensa competencia entre las farmacéuticas por alcanzar la aprobación de la Agencia de Drogas y Alimentos -FDA, por sus siglas en inglés- $y$ atender un potencial mercado de millones de consumidores. La multinacional Pfizer impulsó así su producto para la disfunción eréctil (Viagra), el cual alcanzó, solo en 2001, un volumen de ventas superior a los mil quinientos millones de dólares (Forcades, 2006), lo cual habla no solo de la funcionalidad del medicamento, sino de la extensión de su uso bajo condiciones no diagnosticadas o prescriptas por el personal médico. Según El Espectador (2007), en Colombia el negocio de píldoras para la disfunción eréctil superó los trece millones de dólares por año en 2007 , con un crecimiento en ventas del $20 \%$ anual.

Los productos desarrollados para el tratamiento de la denominada disfunción sexual femenina, son diversos y diferenciados según el trastorno al que son dirigidos. 
Entre ellos se encuentra el Orgasmatrón, invento patentado por el Dr. Stuart Meloy, consiste en implantar un dispositivo eléctrico que estimula la médula espinal y que supuestamente produce un placer instantáneo; también la píldora Alista (Alprostadil), de la farmacéutica Vivus, cuyo agente activo está prescripto para la disfunción eréctil crónica; así como toda clase de geles, parches y preparados de estrógenos y testosterona se presentaron como soluciones milagrosas para las disfunciones sexuales de las mujeres.

Aunque ninguno de estos tuvo aprobación de la FDA como tratamiento efectivo para la DSF, bien por insuficiencia o legitimidad de estudios clínicos o por los efectos secundarios producidos, y dado que algunos están autorizados para otros trastornos, terminan siendo promocionados, recetados y consumidos para la DSF.

Con la elaboración del Sildenafilo en 1992 por Pfizer, y la aprobación en 1998 de la FDA para tratar la disfunción eréctil, se intentó extender su margen de actuación al campo femenino, específicamente para el tratamiento del denominado «trastorno de excitación». El doctor I. Goldstein -señalado como padre del Viagra-, profesor de Urología de la Universidad de Boston, fue el primero en tratar en su clínica las disfunciones sexuales de las mujeres con Sildenafilo, principio activo que posteriormente resultara comercializado sin fórmula médica y sin aprobación de la FDA en algunos países, con los nombres de Lovegra o Kamagra (Viagra femenino).

Dada la complejidad de la sexualidad femenina y los pocos logros experimentales obtenidos en la exploración de fármacos para tratar el trastorno de la excitación, la búsqueda se orientó a los trastornos del deseo, entre ellos el denominado «trastorno de deseo sexual hipoactivo» (TDSH). Esta disfunción, de la cual no hay evidencia contundente de que sea una condición médica real, fue establecida por la industria en el año 2004 con la intención de preparar el mercado para un parche de testosterona para mujeres. Algunos consideran que estas son estrategias de los grandes laboratorios, en la medida que desarrollan una nueva condición para construir el reconocimiento de una necesidad no satisfecha por el mercado (Meixel et al., 2015).

Rosemary Basson, una de las principales investigadoras de la sexualidad femenina, ha criticado el trastorno del deseo sexual hipoactivo, describiéndolo como un diagnóstico «problemático» porque no abarca completamente la comprensión contemporánea de la complejidad de respuestas sexuales de las mujeres (como se cita en Moynihan, 2005).

De acuerdo con el Manual Diagnóstico y Estadístico de los Trastornos Mentales (DSM), el trastorno de deseo sexual hipoactivo se caracteriza por un «persistente o recurrente déficit (o ausencia) de fantasías sexuales y deseo de actividad sexual "que causa" malestar o dificultades interpersonales» (APA, 200o). Dicho trastorno 
fue eliminado en la más reciente edición del DSM, resultando ahora combinado con el término «trastorno de interés sexual/excitación femenina».

Tal como sucede con la mayor parte de las herramientas de diagnóstico de la disfunción sexual femenina, el TDSH se diagnostica en buena medida a partir de cuestionarios desarrollados por los fabricantes de medicamentos, recibiendo críticas tanto en la construcción de las herramientas como en la interpretación que pueda hacerse de las mismas. Por ejemplo, la primera pregunta del Índice de Función Sexual Femenina es: «En las últimas 4 semanas, ¿con qué frecuencia experimentó deseo o interés sexual?» Dan a las mujeres opciones de respuesta que van desde «casi nunca o nunca» hasta «casi siempre o siempre» con puntuaciones que van de 1 a 5 . Una mujer que dice sentir deseo o interés sexual el 50\% de sus horas de vigilia en el último mes, solo alcanzará 3 puntos y estaría al límite para ser diagnosticada con el trastorno (Meixel et al., 2015).

A pesar de que la disminución del interés en el sexo puede ser temporal o causada por las tensiones en el ritmo de vida, enfermedades, uso de medicamentos, problemas de pareja, fatiga, stress $u$ otros factores que necesariamente deben ser tratados por una medicina social o con terapias psicológicas, este desinterés sexual termina en muchos casos siendo diagnosticado como TDSH, lo que conlleva a un tratamiento farmacológico.

Como se mencionó anteriormente, tratamientos desarrollados para mejorar la libido femenina, tales como el parche de testosterona Intrinsa de Procter \& Gamble, la gel de testosterona LibiGel de Biosante Pharmaceuticals, y el Viagra femenino de Pfizer, que fracasaron en su intento de aprobación por la FDA, a pesar de las millonarias inversiones en publicidad y mercadeo realizadas por estas compañías antes de la aprobación, las cuales tenían como destinatarios estratégicos a los profesionales de salud, los periodistas y el público en general (Moynihan, 2005). Sin embargo, y después de dos intentos previos, la Flibanserina logró en agosto de 2015 la aprobación para ser comercializada bajo el nombre de Addyi como primer fármaco para el tratamiento del TDSH. Este medicamento fue desarrollado en principio en el campo psiquiátrico como antidepresivo y resultó refundado para la disfunción sexual femenina. Esta es una práctica recurrente de la industria farmacológica para extender los tiempos de las patentes y ampliar el potencial de mercado de sus productos.

Algunos especialistas han cuestionado la Flibanserina por sus efectos fisiológicos, al señalar que cambia el balance de neurotransmisores cerebrales como la dopamina y la serotonina, advirtiendo sobre efectos secundarios del fármaco tales como baja en la presión sanguínea, náuseas, desmayos, mareos y somnolencia. Para la psiquiatra Julie Holland (2015), el tratamiento con inhibidores selectivos de 
la receptación de serotonina, como es el caso de la Flibanserina, tiene profundos efectos emocionales en la mujer, pues «si los niveles de serotonina son constantemente y artificialmente altos, está en riesgo de perder las fluctuaciones naturales de su sensibilidad natural».

En primera instancia la Flibanserina fue patentada por la multinacional Boehringer Ingelheim, pero tras la primera negativa de aprobación por la FDA la patente fue vendida en 2011 a Sprout Farmaceuticals, la cual, después del segundo fracaso de aprobación en 2013, se encargaría no solo de continuar su desarrollo y generar estudios clínicos para su futura aprobación, sino que además se concentró en lanzar campañas de relaciones públicas con grupos de médicos, asociaciones de mujeres y mujeres congresistas para señalar a la FDA ante la opinión pública como «sexista», por aprobar medicamentos contra la disfunción sexual masculina pero negarse a la aprobación de aquellos destinados a combatir el trastorno en las mujeres (Meixel et al., 2015). A pesar de la oposición y de las dudas creadas en torno a la eficacia del fármaco, su comercialización resultó finalmente aprobada. Al día siguiente se anunció la venta de Sprout Farmaceuticals al grupo farmacéutico Valeant por mil millones de dólares (Clarín, 2015).

Siguiendo a autores como Moynihan, Heat y Henry (2002), el TDSH es un ejemplo de «construcción corporativa de la enfermedad», en la medida que son las farmacéuticas las principales agentes en el patrocinio de la definición de la misma, así como en su promoción para prescriptores y consumidores, estableciendo un estado de enfermedad en la mente de los clínicos y de la población en general años antes de la aprobación y lanzamiento de las drogas, mediante congresos y programas de educación continuada, así como orientando la opinión pública a través de medios masivos de comunicación, en una estrategia de mercadeo denominada «condición de marca» (Meixel et al., 2015).

Si bien los documentos científicos han servido de base para consolidar la disfunción sexual femenina, son los artículos periodísticos, divulgados en los medios masivos, una de las vías por las cuales se afianza e interioriza en la opinión pública. Los medios cuentan con autonomía para posicionar conceptos de salud y se encargan de difundir y consolidar el discurso médico en el conglomerado social favoreciendo los intereses de los grandes laboratorios.

\section{Conclusiones}

Si bien se reconoce que el avance de las tecnologías aplicadas a la salud y el desarrollo de fármacos por la industria ha sido útil para mejorar la calidad y la expectativa 
de vida en los seres humanos, también se debe reconocer que el poder económico, político, médico y social que han adquirido las empresas farmacológicas ha llegado a límites cuestionables.

Todo apunta a considerar que las multinacionales del sector se interesan cada vez menos por la salud de las personas y más por generar estrategias de mercado para sus productos, sean estos nuevos o refundados para patologías recién definidas. En tal sentido, el aumento de la mercantilización y farmaceuticalización de la atención en salud ha llevado a tratamientos médicos más parecidos a los bienes comunes sujetos a las fuerzas del mercado, lo cual nos anima a redefinir la autopercepción, que pasa de paciente pasivo a consumidor activo.

Las reflexiones expuestas en el presente artículo no pretenden negar la existencia de ciertos trastornos que afectan las condiciones de vida de algunas personas, sino demostrar la ampliación de los límites de la enfermedad, en su definición y prevalencia, extendiendo las fronteras entre lo normal y lo patológico; lo cual legitima la intervención médica y consolida la expansión del mercado para beneficio de la industria farmacéutica.

La «promoción de la enfermedad», también llamada «tráfico de enfermedades», «invención de la enfermedad» o «construcción corporativa de la enfermedad», constituye un problema de salud pública por diferentes motivos.

En primer lugar, se está hablando de la posible medicalización de estados «normales» que resultan redefinidos en sus límites y trasmutados artificialmente en condiciones patológicas de gran prevalencia en el conjunto social con el objetivo de crear o ampliar el mercado de fármacos, impulsando el uso excesivo de medicamentos.

De otro lado, la definición de nuevas patologías con el auspicio de los grandes laboratorios trae consigo un gasto innecesario de todo tipo de recursos que podrían ser utilizados en la investigación de patologías de mayor prevalencia o con mayor riesgo para la vida de las personas.

Por último, el señalamiento de algunos investigadores en cuanto a los efectos secundarios y los problemas de salud concomitantes al uso de fármacos prescriptos para las nuevas disfunciones, si bien resultan minimizados o trivializados en los estudios presentados por las farmacéuticas, generan controversia sobre el papel iatrogénico de dichos medicamentos

Algunos investigadores del sexo han cuestionado el concepto de disfunción sexual femenina, ya que resta importancia a factores relacionales y culturales, y sobrevalora los procesos neurofisiológicos como determinantes. Además, debido a la dificultad para distinguir entre una verdadera disfunción y una respuesta adaptativa sana, algunos científicos consideran que cualquier estimación basada en estudios de prevalencia de la enfermedad es poco fiable. 


\section{Referencias}

AGUILAR, M. T. (2010). Descartes y el cuerpo-máquina. Pensamiento, 66 (249), 755-770.

AMERICAN PSYCHIATRIC ASSOCIATION - APA. (2000). Diagnostic and statistical manual of mental disorders. Washington DC: American Psychiatric Press.

ANTONA, A. (2013). Disfunciones sexuales. Notas de clase. Madrid: Instituto de Salud Pública.

APPLBAUM, K. (2006). Pharmaceutical Marketing and the Invention of the Medical Consumer. PLoS Medicine, 3 (4), 23-25.

BAUMAN, Z. (2007). Vida de Consumo. Buenos Aires: Fondo de Cultura Económica.

BÉJIN, A. (1987). Crepúsculo dos psicoanalistas, manhâ dos sexólogos. En Ariés, P. (Coord.) Sexualidades ocidentais. Contribuciôes para a historia e para a sociologia da sexualidade. Sao Paulo: Edit. Brasiliense.

BLECH, J. (2009). Los inventores de enfermedades. Cómo nos convierten en pacientes. España: Ed. Destino.

BULLOUGH, V. (1994). Science in the Bedroom: A History of Sex Research. U.S.A: Basic Books.

CAPRA, F. (1992). El punto crucial. Buenos Aires: Edit. Troquel.

CASINO, G. (25 de abril de 2006). La Promoción de la Enfermedad. El País, Madrid.

CHOMSKY, N., y HERMAN, E. (2009). Los Guardianes de la Libertad. Barcelona: Editorial Crítica.

CONRAD, P. (2005). The shifting engines of medicalization. Journal of Health and

SOCIAL BEHAVIOR, 46 (1), 3-14.

CONRAD, P. \& LEITER, V. (2004). Medicalization, Markets and Consumers. Journal of Health and Social Behavior, 45 (Extra Issue), 158-176.

Disfunción eréctil estimula mercado de \$38 mil millones. (7 de marzo de 2007). El Espectador. Recuperado de: http://www.elespectador.com/impreso/cuadernilloa/negocios/articuloimpreso-disfuncion-erectil-estimula-mercado-de-38-mil-millones

DORAN, E., \& HOGUE, C. (2014). Potency, Hubris and Susceptibility: The Disease Mongering Critique of Pharmaceutical Marketing. The Qualitative Report, 19 (39), 1-18.

FORCADES I VILA, T. (2006). Los crímenes de las grandes compañias farmacéuticas. Barcelona: Els Quaderns de Cristianisme i Justícia, 141.

FOUCAULT, M. (2012). Historia de la sexualidad. La voluntad del saber. México: Siglo XXI.

FOUCAULT, M. (2006). Seguridad, territorio, población. Buenos Aires: Fondo de Cultura Económica. 
FOUCAULT, M. (2003) El nacimiento de la clínica. Una arqueología de la mirada médica. Argentina: Siglo XXI.

HOLLAND, J. (13 de septiembre de 2015). «La mujer moderna tiende a suprimir sus emociones». (Entrevista por Alarcón, A.) El Tiempo. Recuperado de: http://www.eltiempo.com/estilo-de-vi$\mathrm{da} /$ salud/medicamentos-para-controlar-emociones/16371984

ILICH, I. (1975). Némesis Médica. Barcelona: Barral Editores.

ILLOUZ, E. (2007). Intimidades congeladas. Las emociones en el capitalismo. Argentina: Katz Editores.

JONES, D. y COGNA, M. (2012). Sexología, medicalización y perspectiva de género en la Argentina contemporánea. Ciencia, Docencia y Tecnología, 23 (45), 33-59.

LAUMANN, E., PAIK, A. \& ROSEN, R. (2000). Sexual dysfunction in the United States:

PREVALENCE AND PREDICTORS. JAMA, 281 (6), 537-544.

LEXCHIN, J. (2006). Bigger and Better: How Pfizer Redefined Erectile Dysfunction. PLoS Medicine, 3 (4), 7-10.

MARX, K. (1977). El Capital. Tomo I. México D. F.: Fondo de Cultura Económica.

MASTERS, W. y JOHNSON, V. (1972). Entrevista de PLAYBOY: Masters y Johnson. En N. Lehrman, Las técnicas sexuales de Marters y Johnson. Hacia una sexualidad sin problemas. Buenos Aires: Granica Editor.

MEIXEL, A., YANCHAR, E., FUGH-BERMAN, A. (2015). Hypoactive Sexual Desire Disorder: Inventing a Disease to Sell Low Libido. Journal of Medical Ethics, 41 (10), 859-862.

MINTZES, B. (2006). Disease Mongering in Drug Promotion: Do Governments Have a Regulatory Role? PLoS Medicine, 3 (4), ooo1-0oo5.

MOYNIHAN, R. (2005). The Marketing of a Disease: Female Sexual Dysfunction. British Medical Journal, 330 (7484), 192-194.

MOYNIHAN, R., \& HENRY, D. (2006). The Fight Against Disease Mongering: Generating Knowledge for Action. PloS Medicine, 3 (4), pp. ooo1-0004.

MOYNIHAN, R., HEART, I., \& HENRY, D. (2002). Selling Sickness: The Pharmaceutical Industry and Disease Mongering. British Medical Journal, 324 (7342), 886-891.

NATELLA, G. (2008). La creciente medicalización contemporánea: Prácticas que la sostienen, prácticas que la resisten en el campo de la salud mental. En E. Luchtenberg y A. Canelote (Coords). Medicalización y sociedad. Lecturas críticas sobre un fenómeno en expansión. Argentina: SEDRONAR.

PARAMIO, L. (2000). Decisión Racional y Acción Colectiva. Leviatán, (69), 65-83. 
PÉREZ-CIORDIA, I. (2011). Las «no enfermedades» y la medicalización. Jano, 73-76. Recuperado de: http://www.jano.es/ficheros/sumarios/1/oo/1775/73/1voon1775a90034643pdfoo1.pdf

POHL-VALERO, S. (2010) Termodinámica, pensamiento social y biopolítica en la España de la Restauración. Universitas Humanística, 69 (69), 35-60

RAMALHO, V. (2010a). Tecnologias Discursivas na Propaganda de Medicamentos. Discurso y Sociedad, 4 (3), 507-537.

RAMALHO, V. (2010b). Uma Leitura Crítica da Interdiscursividade: O Caso da Publicidade de Medicamento. Caderno de Letras da UFF, (4)o, 117-130.

ROHDEN, F. (2008). O império dos hormonios e a construcao da diferencia entre os Sexos. Historia, Ciencias, Saúde - Manguinhos, 15 (suppl. o), 133-152.

RODRÍGUEZ DÍAZ, S. (2008). El proceso de medicalización y sus consecuencias. Entre la moral, el poder y el negocio. Intersticios. Revista de Sociología del pensamiento crítico, 2, (2). Recuperado de: www.intersticios.es/article/download/2714/2128.

RODRÍGUEZ, P. G. (2015). Alimentación y medicalización. Análisis de un dispositivo de cuidado personal y potenciación de la salud. Revista Sociológica, 86 (30), 201-234.

RODRÍGUEZ, P. G. (2010). La medicalización como estrategia biopolítica. A Parte Rei, (70). Recuperado de: http://serbal.pntic.mec.es/ cmunoz11/zoya7o.pdf

ROSENBERG, C. (1992) Framing Disease: Illness, Society, and History. En Explaining Epidemics and other Studies in the History of Medicine (pp.305-318). Cambridge: Cambridge University Press.

SFEZ, L. (2008). La salud perfecta. Buenos Aires: Prometeo Libros.

SHANKAR, P., \& SUBISH, P. (2007). Disease Mongering. Singapore Medical Journal, 48 (4), 275-280.

TIEFER, L. (2006). Female Sexual Dysfunction: A Case Study of Disease Mongering and Activist Resistance. PLoS Medicine, 3 (4), 14-18.

TREBISACCE, C. (2015). Discursos científicos sobre la sexualidad femenina y la respuesta de las feministas y los varones homosexuales en la década del sesenta en Argentina. Sexualidad, Salud y Sociedad, 20, 49-71.

Viagra femenino: venden en US\$1.00o millones el laboratorio que lo patentó. (2015, 20 de agosto). Clarín. Recuperado de: http://www.clarin.com/sociedad/Viagra- femenino-US-millones-laboratorio_o_1415858644.html 